Supporting information for:

\title{
From bifunctional nucleophilic behavior of DBU to a new fluorescent heterocyclic
}

\author{
platform
}

Daniel T. Gryko, Joanna Piechowska, Mariusz Tasior, Jacek Waluk and Grażyna Orzanowska

\section{Table of Contents}

1. Experimental procedures and references

2. Proposed mechanism of 2-amino-pyrrolo[2,3-b]quinoxalines formation

3. Packing of crystals for compound $\mathbf{3}$

4. Spectral data
S1-S2

S3

S4

S5

\section{Experimental procedures and references Synthesis}

All chemicals were used as received unless otherwise noted. All reported ${ }^{1} \mathrm{H}$ NMR and ${ }^{13} \mathrm{C}$ NMR spectra were recorded on $500 \mathrm{MHz}$ spectrometer. Chemical shifts $(\delta \mathrm{ppm})$ were determined with TMS as the internal reference; $J$ values are given in Hz. UV-Vis spectra were recorded in acetonitrile. Chromatography was performed on silica (Kieselgel 60, 200-400 mesh). Mass spectra were obtained via EI. The purity of new compounds was established based on NMR spectra and elemental analysis.

2,3,4,5,6,7-hexahydro-1H-3a,8,13,13b-tetraazabenzo[b]cyclohepta[1,2,3-jk]fluorene (3). 2,3-Dichloroquinoxaline $(0.5 \mathrm{~g}, 2.5 \mathrm{mmol})$ and 1,8-diazabicyclo[5,4,0]undec-7-ene $(1.12 \mathrm{ml}$, $7.5 \mathrm{mmol}$ ) were heated at $150^{\circ} \mathrm{C}$ for $45 \mathrm{~min}$. Subsequently, the warm residue was dissolved in $\mathrm{MeOH}$ and cooled down. This solution was poured into the water and precipitated dark solid was filtered and discarded. Then acetic acid $(10 \mathrm{ml})$ was added to the water solution and the mixture was extracted with $\mathrm{CH}_{2} \mathrm{Cl}_{2}(4 \times 50 \mathrm{ml})$. Water fraction was neutralized with $\mathrm{NaHCO}_{3}$ and extracted with $\mathrm{CH}_{2} \mathrm{Cl}_{2}$ again. The last organic extracts was washed with water, dried $\left(\mathrm{Na}_{2} \mathrm{SO}_{4}\right)$ and evaporated. The pure product was obtained after crystallization $\left(\mathrm{CH}_{2} \mathrm{Cl}_{2} / \mathrm{Et}_{2} \mathrm{O}\right),(0.25 \mathrm{~g}, 35 \%)$. M.p. $=167-168{ }^{\circ} \mathrm{C} .{ }^{1} \mathrm{H} \mathrm{NMR}\left(500 \mathrm{MHz}, \mathrm{CDCl}_{3}, \delta\right) 1.81-1.90$ $(\mathrm{m}, 2 \mathrm{H}), 1.95-2.03(\mathrm{~m}, 2 \mathrm{H}), 2.22-2.30(\mathrm{~m}, 2 \mathrm{H}), 2.99(\mathrm{t}, 2 \mathrm{H}, J=6 \mathrm{~Hz}), 3.37(\mathrm{t}, 2 \mathrm{H}, J=5.5$ $\mathrm{Hz}), 3.42(\mathrm{t}, 2 \mathrm{H}, J=5.5 \mathrm{~Hz}), 4.20(\mathrm{t}, 2 \mathrm{H}, J=6 \mathrm{~Hz}), 7.38-7.46(\mathrm{~m}, 1 \mathrm{H}), 7.46-7.53(\mathrm{~m}, 1 \mathrm{H})$, 7.90-7.96 (m, 1H), $8.02(\mathrm{~d}, 1 \mathrm{H}, \mathrm{J}=8 \mathrm{~Hz}) .{ }^{13} \mathrm{C} \mathrm{NMR}\left(500 \mathrm{MHz}, \mathrm{CDCl}_{3}, \delta\right)$ 22.0, 23.2, 27.1, 29.9, 38.1, 49.9, 56.4, 90.2, 124.2, 125.5, $2 \times 127.4,137.1,141.8,144.0,152.1$. EI-HR obsd 278.1546 $\left[\mathrm{M}^{++}\right]$, calcd exact mass $278.1532\left(\mathrm{C}_{17} \mathrm{H}_{18} \mathrm{~N}_{4}\right)$. Anal. Calcd for $\mathrm{C}_{17} \mathrm{H}_{18} \mathrm{~N}_{4}$ : C, 73.35; H, 6.52; N, 20.13. Found: C, 73.15; H, 6.47; N, 19.99. $\lambda_{\text {abs }}\left(\right.$ acetonitrile $\left.\varepsilon \times 10^{-3}\right) 279$ ( 37.3 ), $350(12.3), 418(12.4) \mathrm{nm}$.

2,3,4,5,6,7-hexahydro- $1 H$-3a,8,11,11b-tetraazacyclohepta[1,2,3-jk]fluorene (4). 2,3Dichloropyrazine $(300 \mathrm{mg}, 2 \mathrm{mmol})$ and 1,8-diazabicyclo[5,4,0]undec-7-ene $(900 \mu \mathrm{l}, 6$ 
mmol) were heated at $110^{\circ} \mathrm{C}$ for $1 \mathrm{~h}$. Subsequently, the warm residue was dissolved in $\mathrm{MeOH}$ and cooled down. The $\mathrm{MeOH}$ was partially evaporated and chromatographed (silica, $\left.\mathrm{CH}_{2} \mathrm{Cl}_{2} / \mathrm{MeOH}, 99: 1\right)$ to obtain pure product $(0.19 \mathrm{~g}, 42 \%)$. M.p. $=95{ }^{\circ} \mathrm{C} .{ }^{1} \mathrm{H}$ NMR $(500 \mathrm{MHz}$, $\left.\mathrm{CDCl}_{3}, \delta\right) 1.76-1.81(\mathrm{~m}, 2 \mathrm{H}), 1.91-1.96(\mathrm{~m}, 2 \mathrm{H}), 2.20-2.25(\mathrm{~m}, 2 \mathrm{H}), 2.89(\mathrm{t}, 2 \mathrm{H}, J=6 \mathrm{~Hz})$, $3.24(\mathrm{t}, 2 \mathrm{H}, J=5.5 \mathrm{~Hz}), 3.35(\mathrm{t}, 2 \mathrm{H}, J=5.6 \mathrm{~Hz}), 4.13(\mathrm{t}, 2 \mathrm{H}, J=6 \mathrm{~Hz}), 7.77(\mathrm{~d}, 1 \mathrm{H}, \mathrm{J}=2.97$ $\mathrm{Hz}), 8.09$ (d, 1H, J = 2.97 Hz). ${ }^{13} \mathrm{C}$ NMR $\left(500 \mathrm{MHz} \mathrm{CDCl}_{3}, \delta\right) \quad 22.1,23.1,27.3,30.4,38.2$,

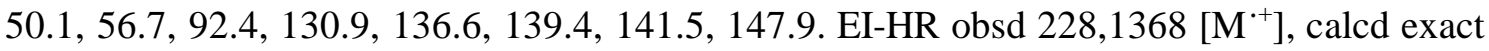
mass $228.1375\left(\mathrm{C}_{17} \mathrm{H}_{18} \mathrm{~N}_{4}\right)$. $\lambda_{\text {abs }}$ (acetonitrile $\left.\varepsilon \times 10^{-3}\right) 261$ ( 12.8 ), 312 ( 4.1 ), 370 ( 7.7$) \mathrm{nm}$.

\section{Optical measurements}

Electronic absorption spectra were measured on a Shimadzu UV 3100 spectrophotometer. Stationary fluorescence spectra were recorded and corrected for instrumental response using an Edinburgh FS 900 CDT spectrofluorometer. The solvents: $n$-hexane, DMSO, and methanol were checked for the presence of fluorescent impurities. For the determination of quantum yields, quinine sulfate in $1 \mathrm{~N} \mathrm{H}_{2} \mathrm{SO}_{4}$ was used as a standard $(\varphi=0.55)$. Fluorescence lifetimes were obtained using an Edinburgh FL 900 CDT time-resolved fluorometer, with estimated time resolution of 300 ps. Nitrogen flash lamp was used as the excitation source. Both samples were excited at $337 \mathrm{~nm}$.

\section{Crystal data for compound 3.}

Crystal data for 3: $\mathrm{C}_{17} \mathrm{H}_{18} \mathrm{~N}_{4}, \mathrm{M}=278.35$, brown prism, $0.40 \times 0.30 \times 0.25 \mathrm{~mm}$, monoclinic, space group $P 2{ }_{1} / c$ (No. 14), $a=7.4562(12), b=16.273(3), c=11.316(2) \AA, \beta=95.83(10)^{\circ}, V$ $=1365.9 \AA^{3}, Z=4, D_{\mathrm{c}}=1.354 \mathrm{~g} / \mathrm{cm}^{3}, F_{000}=592$, MoK $\alpha$ radiation, $\lambda=0.71073 \AA$, $T=$ $100(2) \mathrm{K}, 2 \theta_{\max }=44.9^{\circ}, 3349$ reflections collected, 1756 unique $\left(\mathrm{R}_{\mathrm{int}}=0.0507\right)$. The structure was solved and refined using the programs SHELXS- $97^{1}$ and SHELXL-97 ${ }^{2}$ respectively. Final $G o o F=0.960, R 1=0.0691, w R 2=0.1690, R$ indices based on 955 reflections with $\mathrm{I}$ I>2(I) (refinement on $F^{2}$ ), 190 parameters, 0 restraints. Lp and absorption corrections applied, $\mu=0.084 \mathrm{~mm}^{-1}$.

\section{References:}

(1) G. M. Sheldrick, Acta Crystallogr., Sect A 1990, 46, 467.

(2) G. M. Sheldrick, University Göttingen, Germany, 1997. 
Scheme S1. Proposed mechanism of 2-amino-pyrrolo[2,3-b]quinoxalines formation.

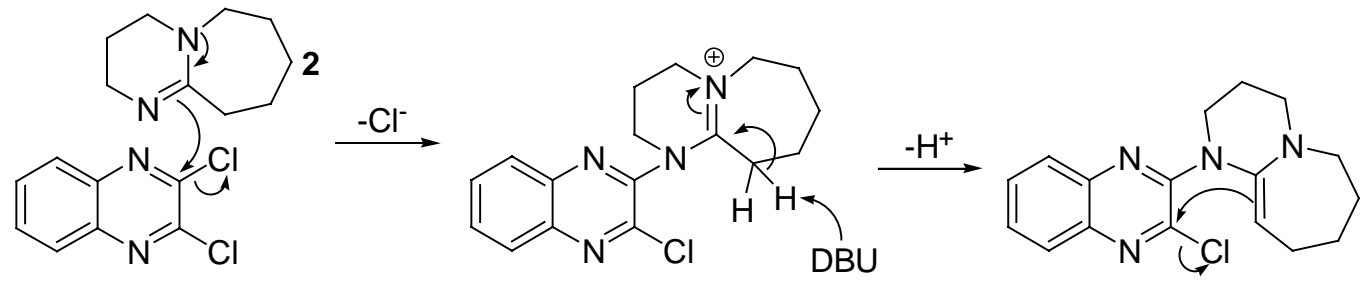

1

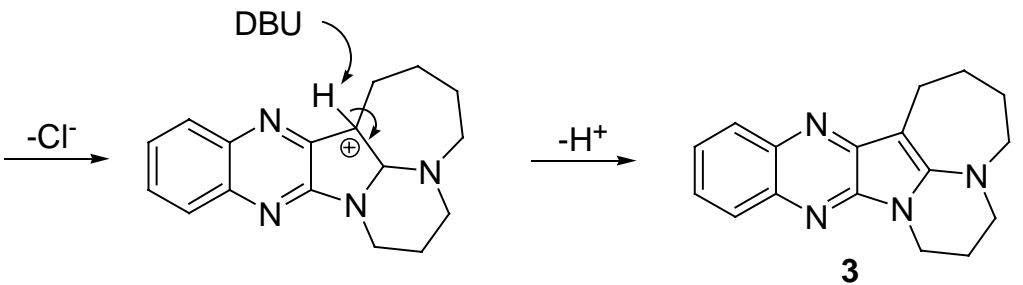


Figure S1. Packing of crystals for compound 3.
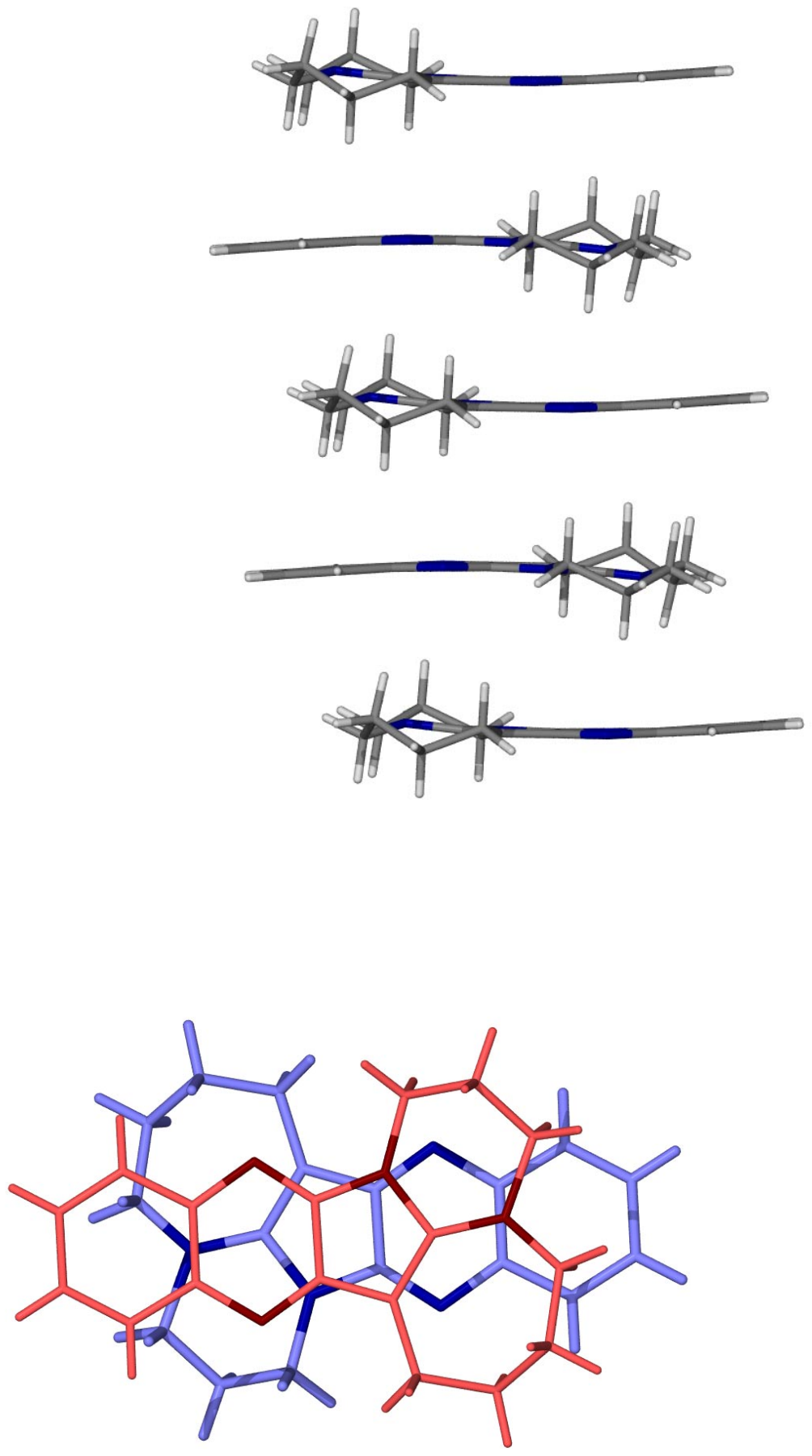


\section{Spectral data}
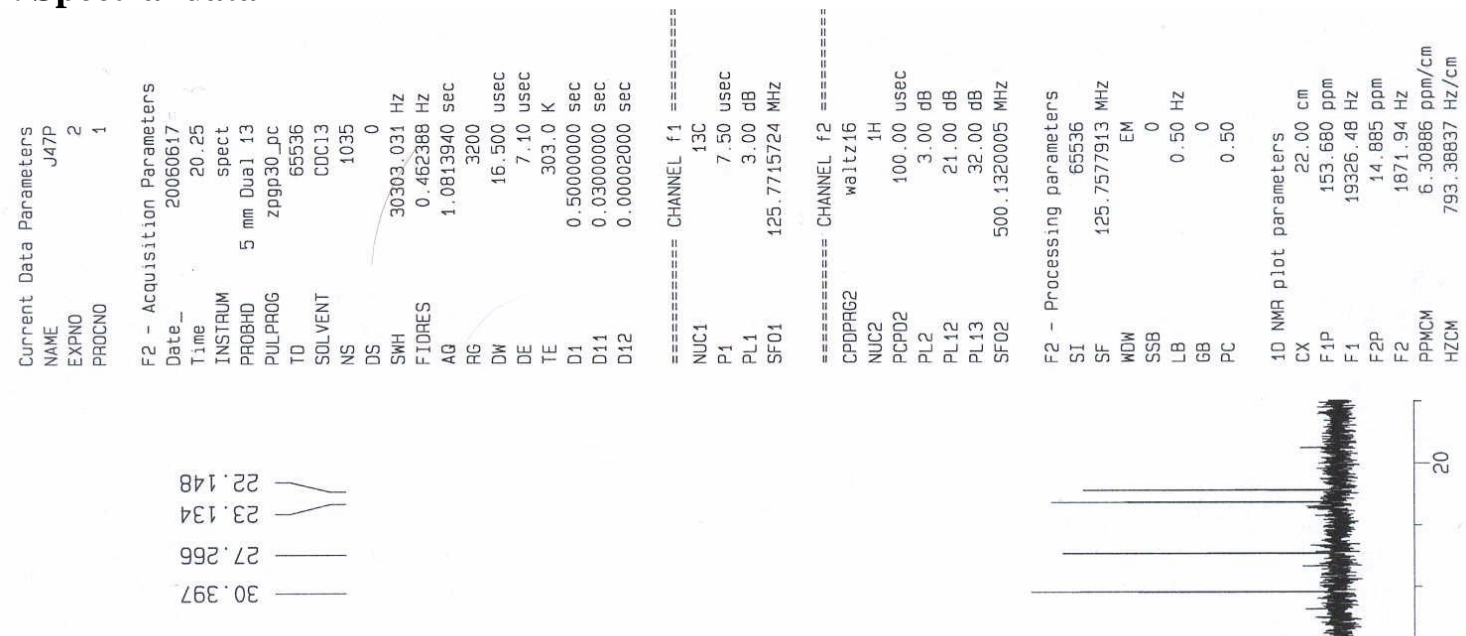

$902 \cdot 8 E$

$\angle 00^{\circ}$ it

$890^{\circ} 09^{-}$

$9 \angle 9 \cdot 99$

$\angle \nabla L 9 \angle$

$098 \cdot 9 L$

$988^{\circ} 92$

GSट $L L$

ट૬E ¿6

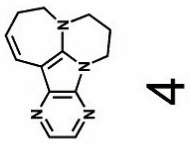

$\varepsilon \angle 8^{\circ} 0 \varepsilon \digamma-$

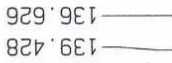

$\hookrightarrow \angle \nabla^{\circ} \downarrow \nabla レ$

$\angle G^{\circ} \angle \nabla I$

wdd

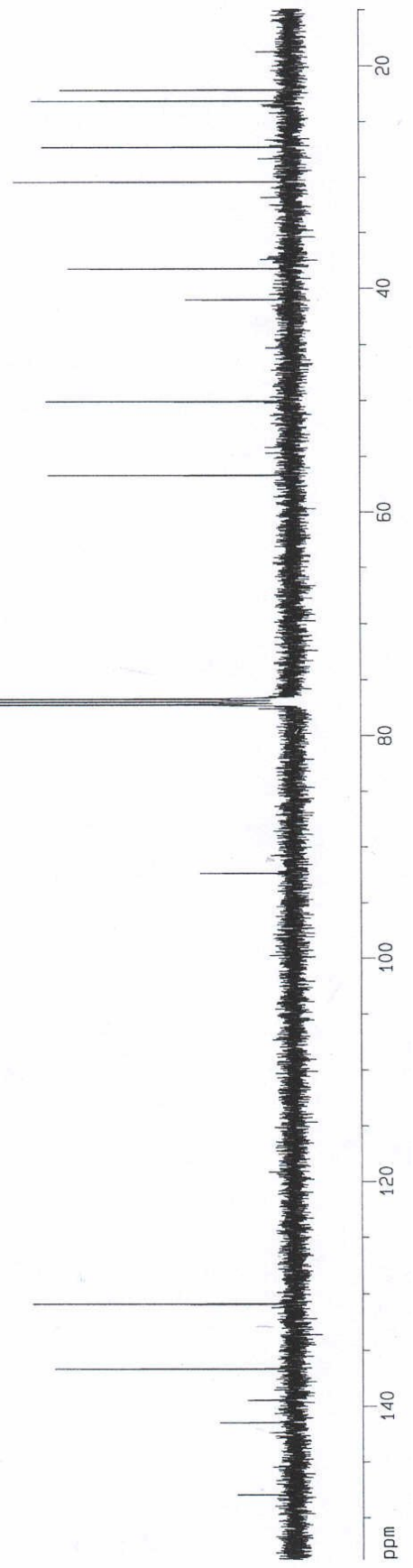

